\title{
Overexpression of Numb suppresses tumor cell growth and enhances sensitivity to cisplatin in epithelioid malignant pleural mesothelioma
}

\author{
YANMENG KANG ${ }^{1}$, MINGJIE DING ${ }^{2}$, GUANGYAN TIAN $^{3}$, HAISHENG GUO $^{4}$,

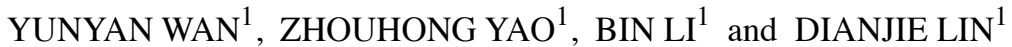

\author{
${ }^{1}$ Department of Respiratory Medicine, Shandong Provincial Hospital, Shandong University, Jinan, \\ Shandong 250021; Departments of ${ }^{2}$ Respiratory Medicine, and ${ }^{3}$ Neurology, Jinan Children's Hospital, Jinan, \\ Shandong 250022; ${ }^{4}$ Department of Oncology, Dongying People's Hospital, Dongying 257091, P.R. China
}

Received March 2, 2013; Accepted April 11, 2013

DOI: 10.3892/or.2013.2429

\begin{abstract}
Malignant pleural mesothelioma (MPM) is a highly aggressive and conventional treatment-resistant tumor with a dismal prognosis. Among the three histological subtypes of MPM, the epithelioid is the most common type. Numb is considered as a tumor suppressor playing a critical role in controlling asymmetric cell division, maintenance of stem cell compartments, ubiquitination of specific substrates and regulating Notch-, Hedgehog- and TP53-activated pathways. The present study was designed to analyze the role of Numb in epithelioid MPM. We investigated the expression of Numb in 39 epithelioid MPM and 22 normal pleural tissues by immunohistochemistry. Furthermore, we overexpressed Numb in NCI-H2452, an epithelioid human MPM cell line, and investigated the effect of Numb overexpression on the proliferation, apoptosis and sensitivity to cisplatin in cells. The expression of Numb was significantly lower in MPM compared to the control group and Numb had an inverse correlation with the Ki-67 labeling index. Loss of Numb expression was associated with poor prognosis in epithelioid MPM. Overexpression of Numb in NCI-H2452 cells significantly inhibited proliferation, promoted apoptosis and enhanced sensitivity to cisplatin. Moreover, Numb overexpression activated caspase-9 and caspase- 3 through release of cytochrome $c$ as well as downregulation of XIAP and survivin. We speculate that cytochrome $c /$ caspase signaling is a possible mechanism through which Numb enhances the apoptosis of NCI-H2452 cells. These results suggest that Numb may be involved in epithelioid MPM development, and its upregulation may confer sensitivity to cisplatin, suggesting potential therapeutic options for MPM.
\end{abstract}

Correspondence to: Dr Dianjie Lin, Department of Respiratory Medicine, Shandong Provincial Hospital, Shandong University, No. 324 Jing 7 Wei 5 Road, Jinan, Shandong 250021, P.R. China E-mail: lin_dianjie@126.com

Key words: malignant pleural mesothelioma, epithelioid, Numb, overexpression, growth, sensitivity, cisplatin

\section{Introduction}

Malignant pleural mesothelioma (MPM) is a highly aggressive and conventional treatment-resistant tumor with a dismal prognosis. Its incidence has been increasing over the past two decades in industrialized countries and is expected to peak during the period 2010-2020 $(1,2)$. There are three distinct histologic subtypes of MPM and the epithelioid type is the most common one which comprises 50-70\% of all cases. Asbestos exposure, non-asbestos mineral fibers, organic chemicals, radiation and simian virus 40 (SV40) have been suggested as risk factors for mesothelioma (3). These etiologic agents can cause chronic tissue injury and tissue repair in the long latency period. The misappropriating homeostatic mechanisms that govern tissue repair and stem cell self-renewal may lead to carcinogenesis (4). Targeting the genes which play a part in the determination and maintenance of the stem cell compartment may contribute to identifying more accurate prognostic factors and developing effective therapies.

Numb is an evolutionarily conserved protein that plays a critical role in cell fate determination. Numb was originally identified in Drosophila (5). It has been reported to be involved in the control of asymmetric cell division (6), maintenance of stem cell compartments (7), regulation of cell polarity and adhesion (8), migration (9) and ubiquitination of specific substrates (10-12). Subversion of Numb has been linked to many pathological mechanisms, including cancer. Numb plays an important role in the transformation target and the plasticity of the stem cell compartment (13), which are closely related to tumor formation. In particular, the plasticity of the stem cell compartment is related to epithelial-mesenchymal transition (EMT), which contributes to the acquirement of invasive properties as well as resistance to cell apoptosis and chemotherapy $(14,15)$. Subversion of Numb is also predicted to have a major impact on the homeostasis of endocytosis $(16,17)$ and the alteration of many polarity functions through connecting with the PAR complex $(18,19)$, which both facilitate transformation events of tumors. In the absence of Numb, both Notch $(10,20,21)$ and Hedgehog (Hh) signaling (11) are augmented, with pro-proliferative 
and anti-differentiation effects, while the suppressor TP53 signaling is attenuated (12).

Experimental evidence indicates that Numb has the potential to function as a tumor suppressor. Studies of mutant Numb in Drosophila have shown neuroblast overproliferation and tumor formation, which strongly supports the hypothesis that impairment of asymmetric cell division in stem cells results in an imbalance in self-renewal and differentiation which leads to cancer (22). In addition, in vivo RNA interference of Numb in a model of mouse lymphomagenesis was found to accelerate the onset of lymphomas (23). Loss of Numb expression has also been reported in some types of human cancers, such as breast cancers, non-small cell lung carcinomas, salivary gland carcinomas and medulloblastomas $(20,21,24,25)$. In human astrocytomas, there is a trend of higher Numb expression in more malignant tumors (26). Whether Numb functions as a tumor suppressor in MPM or not has not been clarified.

In the present study, we analyzed the expression of Numb in epithelioid MPM and investigated the potential role of Numb in MPM cells. The aim of our study was to clarify the role of Numb in epithelioid MPM and to explore novel strategies for drug-resistant MPM therapy.

\section{Materials and methods}

Patients and specimens. Tissue samples of 39 MPM patients who underwent medical thoracoscopy procedures (Department of Respiratory Medicine, Provincial Hospital, Shandong University, China), from 2007 to 2010, were analyzed and 22 normal pleural samples as a control group were recruited. The MPM diagnosis was based on WHO criteria (27) and confirmed in all instances by clinical, morphologic and immunohistochemical data. Specimens were reviewed by two pathologists, and the histological type was confirmed as epithelioid cell type according to the 2004 WHO classification of pleural tumors (27). All procedures were approved by the Ethics Committee of Shandong University.

Immunohistochemistry. Formalin-fixed and paraffinembedded tissue sections $(4 \mu \mathrm{m})$ were processed for immunohistochemical investigation. Tissue sections were first deparaffinized, followed by rehydration with serially decreased concentrations of ethanol. The following antigen retrieval was carried out in $0.01 \mathrm{M}$ citrate buffer $\left(\mathrm{pH} \mathrm{6.0)}\right.$ ) at $96^{\circ} \mathrm{C}$ for $15 \mathrm{~min}$ in a thermostat controlled water bath. The tissue sections were then immersed in $3 \% \mathrm{H}_{2} \mathrm{O}_{2}$ for $30 \mathrm{~min}$ and were blocked with normal serum at $37^{\circ} \mathrm{C}$ for $30 \mathrm{~min}$. The primary antibodies antiNumb antibody (1:200, rabbit polyclonal; Abcam, Cambridge, UK) and anti-Ki-67 antibody (1:150, rabbit polyclonal; Santa Cruz Biotechnology, Santa Cruz, CA, USA) were incubated at $4^{\circ} \mathrm{C}$ overnight. The following biotinylated secondary antibodies (Beijing Zhongshan Golden Bridge Biotechnology Co., Ltd., Beijing, China) were added at $37^{\circ} \mathrm{C}$ for $30 \mathrm{~min}$. For detection of the binding sites, a streptavidin-biotin system (Beijing Zhongshan Golden Bridge Biotechnology) was used. Diaminobenzidine (DAB) was used as the enzyme substrate to observe the specific antibody localization, and nuclei were counterstained using hematoxylin. Negative controls were performed in all cases by omitting the primary antibodies. Observation was carried out under a light microscope (Leica
DM4000, Wetzlar, Germany) and the images were processed with image processing software (Leica IM45).

Histological evaluation. Two independent observers blinded to the clinical data were assigned to evaluate all samples. Quantitation of cells staining positivity for Numb in the cytoplasm or membrane was recorded. Sections with staining in $\geq 10 \%$ of cells were considered as positive. Immunohistochemical reactivity was graded on a scale of 0-3 according to the percentage of immunopositive cells as follows: grade 0 , no staining or $<10 \%$; grade $1,10-30 \%$; grade 2, 30-50\%; grade 3, $>50 \%$ positive cells. For Ki-67, the percentage of positively stained nuclei out of the total cancer cells was calculated, and we used $25 \%$ as the cut-off point for categorizing.

Cell culture and plasmid transfection. Human MPM cell line NCI-H2452 was stemed from the American Type Culture Collection (ATCC, Manassas, VA, USA), cultured in RPMI-1640 medium supplemented with $10 \%$ fetal calf serum, $1.5 \mathrm{~g} / 1 \mathrm{NaHCO}_{3}, 2.5 \mathrm{~g} / 1$ glucose, $0.11 \mathrm{~g} / 1$ sodium pyruvate and maintained at $37^{\circ} \mathrm{C}$ in a humidified atmosphere with $5 \% \mathrm{CO}_{2}$. We selected the HEK-293T cell line which has been proven to express Numb as a control (11). HEK-293T cells were cultured in Dulbecco's modified Eagle's medium (DMEM) containing $10 \%$ fetal bovine serum (FBS). Plasmid pcDNA3.1-Numb was synthesized by Shanghai GenePharma Co., Ltd. (Shanghai, China). First, $1 \times 10^{6}$ cells in a $60-\mathrm{mm}$ dish were transfected with either the empty vector (mock) or pcDNA3.1-Numb (Numb) using Lipofectamine ${ }^{\mathrm{TM}} 2000$ (Invitrogen) according to the manufacturer's guidelines. After $24 \mathrm{~h}$, the cells were split into three sets: one set was used for cell viability assay; one was used to analyze protein expression by western blot analysis; and one was used for cell apoptosis analysis.

Real-time quantitative reverse transcription-polymerase chain reaction. Primer sequences used for Numb were: 5'-CAATCTCCTACCTTCCAAGGG-3' and 5'-CGGACGC TCTTAGACACCTC-3'; and for GAPDH, 5'-TGGTCACCA GGGCTGCTT-3' and 5'-AGCTTCCCGTTCTCAGCCTT-3'. All primers were synthesized by Takara Co., Ltd. (Dalian, China).

Total RNA from cells was isolated using TRIzol (Invitrogen) and reverse-transcribed by the ReverTra Ace qPCR RT kit (Toyobo, Osaka, Japan). qRT-PCR was performed using SYBR Green Real-Time PCR Master Mix (Toyobo) following the manufacturer's instructions and on the LightCycler 480 System (Roche, Germany). Each reaction was performed in triplicate and analysis was performed by the $2^{-\Delta \Delta \mathrm{Ct}}$ method.

Western blot analysis. At 48-72 h after transfection, cells were harvested for western blot analysis. Total protein was extracted by RIPA lysis buffer. To analyze cytochrome $c$ expression, cytosolic and mitochondrial fractions were prepared using the Mitochondria Extraction kit (Nanjing KeyGen Biotech., Co., Ltd., Nanjing, China). Proteins were transferred to a PVDF membrane and subjected to the antibodies including Numb (1:1,000; Abcam), caspase-3, caspase-9, cytochrome $c$, XIAP and survivin (1:500; Santa Cruz Biotechnology). Peroxidase-labeled anti-mouse or anti-rabbit antibodies were 
used as secondary antibodies (Zhongshan Goldenbridge Biotechnology). Blots were developed using enhanced chemiluminescence detection reagent (Applygen Technologies Inc., Beijing, China).

Cell viability assay. We used an MTT (3-[4,5-dimethylthiazol2-yl]-2,5-diphenyltetrazolium bromide) assay to evaluate cell viability. Cells $\left(5 \times 10^{3} /\right.$ well) were grown in a 96 -well plate and were tested at 24,48 and $72 \mathrm{~h}$ after transfection. The cells (1x10 $/$ well) were grown in a 96-well plate and incubated for another $24 \mathrm{~h}$, and these cells were then treated with different concentrations of cisplatin ranging from 1 to $10 \mu \mathrm{mol} / 1$ for $24 \mathrm{~h}$ in triplicate. Twenty microliters of $5 \mathrm{mg} / \mathrm{ml}$ MTT was added into each well and incubated for $4 \mathrm{~h}$ at $37^{\circ} \mathrm{C}$ in a culture hood. Media were carefully removed, and $150 \mu 1$ DMSO was added. The absorbance was measured at a wavelength of $490 \mathrm{~nm}$ from which the background was subtracted. The cell survival value index was calculated by the formula: [A490 (+cisplatin)/A490 (-cisplatin)] x 100\%.

Cell apoptosis analysis. The morphological changes in apoptotic cells were investigated using Hoechst 33258 staining (Solarbio, Shanghai, China) and fluorescence microscopy. The apoptosis rate was examined by two-color analysis of Annexin V/FITC binding and propidium iodide (PI) uptake using flow cytometry. Cells $\left(1 \times 10^{5}\right)$ were seeded into 6-well plates and were tested at $72 \mathrm{~h}$ after transfection. Cells $\left(1 \times 10^{5}\right)$ were then seeded into 6-well plates and incubated for another $24 \mathrm{~h}$, and these cells were then treated with $5 \mu \mathrm{mol} / \mathrm{l}$ cisplatin for $24 \mathrm{~h}$. FITC-conjugated Annexin V was added at a concentration of $0.5 \mu \mathrm{g} / \mathrm{ml}$. After incubation for $20 \mathrm{~min}$ at room temperature in the dark, PI was added at $1 \mu \mathrm{g} / \mathrm{ml}$, and the samples were immediately analyzed by fluorescence-activated cell sorting (FACS).

Statistical analysis. All analyses were performed using SPSS 17.0 software package (SPSS Inc., Chicago, IL, USA). Chi-square test was used to compare the expression of Numb in the MPM and control groups. Correlations of protein expression (grade scores) with clinical and pathological parameters of the tumors were evaluated with the Mann-Whitney test for ordinal variables. Overall survival (OS) was defined as the time of diagnosis to the time of death from cancerrelated cause or to the date the patient was last known to be alive. The univariate analysis of OS was carried out by the Kaplan-Meier method using the log-rank test. All cell culture experiments were conducted at least three times. Data are shown as means \pm SD and were analyzed using the Student's t-test for two samples. All statistical tests were two-sided, and probability values $<0.05$ and $<0.01$ were defined as being statistically significant.

\section{Results}

Expression of Numb in MPM tissues. The characteristics of the 39 epithelioid MPM patients enrolled in the present study were as follows. The median age for the patients was 55 years (range, 32-77). Nineteen patients were males and 20 were females, with a male to female ratio of 1:1.05. Patients were divided into two groups according to the Eastern Cooperative
Table I. Expression of Numb in epithelioid MPM and its correlation with clinical features.

\begin{tabular}{lccc}
\hline & \multicolumn{2}{c}{$\begin{array}{c}\text { Numb } \\
\text { expression }\end{array}$} & \\
\cline { 3 - 3 } & N & Grade 0/1/2/3 & P-value \\
Variables & 39 & $20 / 10 / 6 / 3$ & \\
\hline MPM (epithelioid) total & & & 0.783 \\
Age (years) & 20 & $10 / 5 / 3 / 2$ & \\
$\quad \leq 55$ & 19 & $10 / 5 / 3 / 1$ & \\
$>55$ & & & 0.258 \\
Gender & 19 & $8 / 5 / 5 / 1$ & \\
$\quad$ Male & 20 & $12 / 5 / 1 / 2$ & \\
$\quad$ Female & & & 0.159 \\
ECOG performance status & 11 & $4 / 3 / 2 / 2$ & \\
$\quad \leq 2$ & 28 & $16 / 7 / 4 / 1$ & \\
$>2$ & & & 0.011 \\
Ki-67 index & & & \\
$\quad \leq 25$ & 26 & $10 / 7 / 6 / 3$ & \\
$>25$ & 13 & $10 / 3 / 0 / 0$ & \\
\hline
\end{tabular}
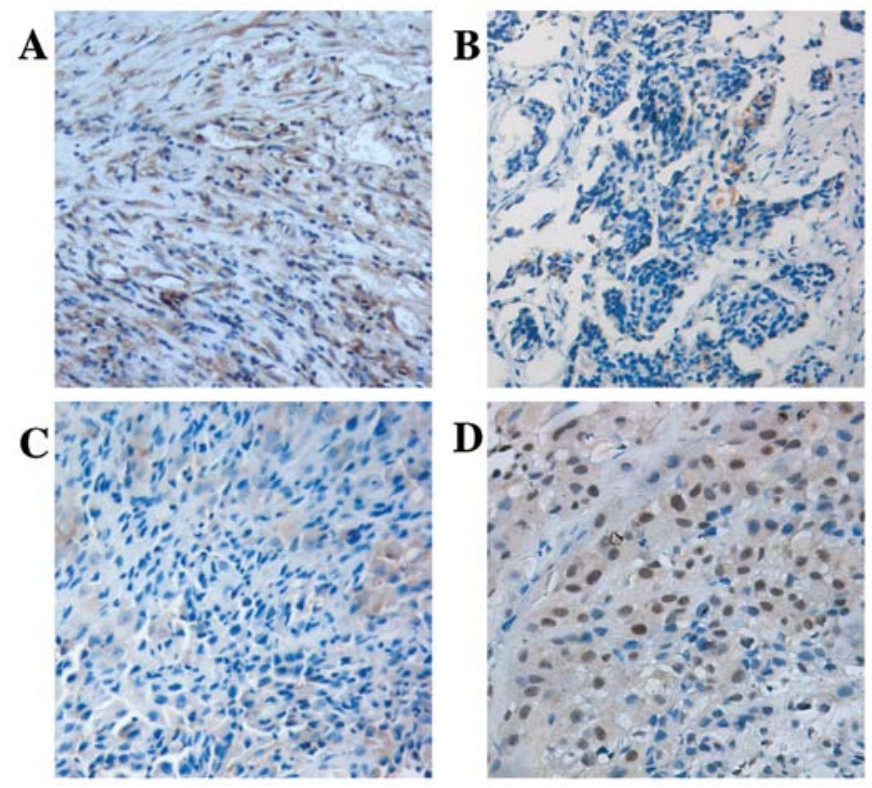

Figure 1. Immunostaining of Numb and Ki-67. (A) Strong positive staining in the control group compared to (B) negative staining of Numb in epithelioid MPM. (C) Representative weak staining of Numb in MPM and the corresponding high Ki-67 labeling index (>25\%); (D), magnification, x200.

Oncology Group (ECOG) performance status. Twenty-nine patients were in the grade $<2$ group and 10 patients were in the grade $\geq 2$ group (Table I).

Numb localization on the membrane or in the cytoplasm was demonstrated in tumor samples [51.3\% (20/39) positive] with a slighter intensity compared with $[86.4 \%(19 / 22)]$ the normal pleural specimens ( $\mathrm{P}<0.05$; Fig. 1A and B). A marked inverse correlation was found between Numb expression levels and Ki-67 labeling index $(\mathrm{P}<0.05$; Fig. $1 \mathrm{C}$ and $\mathrm{D}$, Table I). There was no correlation between loss of Numb 


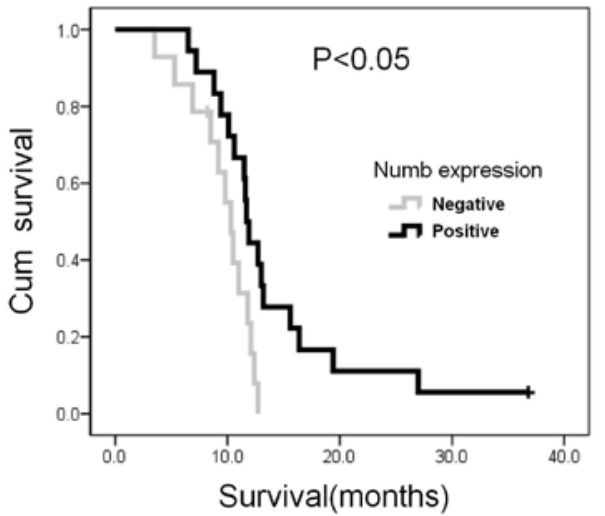

Figure 2. Kaplan-Meier curves showing the overall survival rates according to Numb expression. Numb negativity (grey line) vs. Numb positivity (weak, moderate, strong) (black line).

expression and gender, age and ECOG performance status ( $\mathrm{P}>0.05)$.

Complete clinical follow-up data were obtained from 32 patients allowing retrospective survival analysis. At the completion of the study in July 2011, 30 patients had died, with a median follow-up of 11.3 months. Univariate analysis indicated that overall survival was influenced by Numb expression (log-rank test $\mathrm{P}<0.05$; Fig. 2), comparing negativity (grade 0; median survival, 10.3 months; 95\% CI, 9.1-11.5) vs. weak-moderate-strong expression (grade 1-3; median survival, 11.7 months; 95\% CI, 11.1-12.3).

Numb protein expression is increased in NCI-H2452 cells by transfection. Before transfection, NCI-H2452 cells displayed levels of Numb mRNA expression comparable to that detected in HEK-293T cells, but low protein expression was noted (Fig. 3A and B). The NCI-H2452 cells transfected with pcDNA3.1-Numb showed higher Numb protein expression compared with the mock-transfected control (Fig. 3C).

Numb inhibits proliferation and promotes apoptosis in the NCI-H2452 cells. The absorbance of Numb-transfected cells, as indicated by MTT, was markedly lower at 48 and $72 \mathrm{~h}$ compared to that of the mock-transfected cells $(\mathrm{P}<0.05$, $\mathrm{P}<0.01$; Fig. 4A), which indicates that Numb overexpression inhibited the growth of NCI-H2452 cells. There were no significant differences between the two groups at $24 \mathrm{~h}$ $(\mathrm{P}>0.05)$. Apoptosis was investigated at $72 \mathrm{~h}$ after transfection. Hoechst staining showed that Numb overexpression increased the number of apoptotic cells, which were characterized by several unique morphological nuclear changes, such as chromatin condensation and nuclear fragmentation (Fig. 5). The apoptosis rate was assayed using flow cytometry. The percentage of early and late apoptotic cells was notably higher in the Numb-transfected $(19.88 \pm 3.31 \%)$ when compared to the percentage in the mock-transfected cells (9.41 $\pm 2.34 \%, \mathrm{P}<0.05$; Fig. 4B and C).

Overexpression of Numb induces activation of caspase-3 and caspase-9, as well as cytochrome c release and $X I A P$ and survivin degradation. At 48-72 $\mathrm{h}$ after transfection, we analyzed various proteins related to apoptosis in the
A

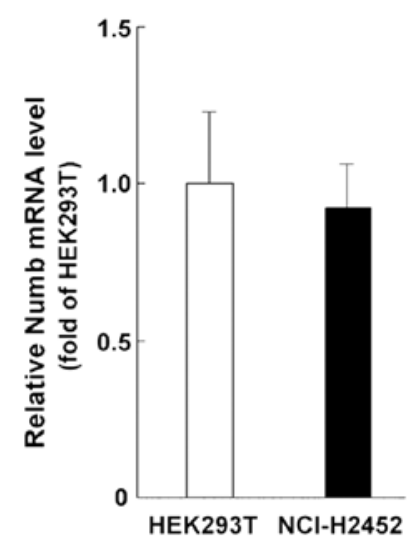

B

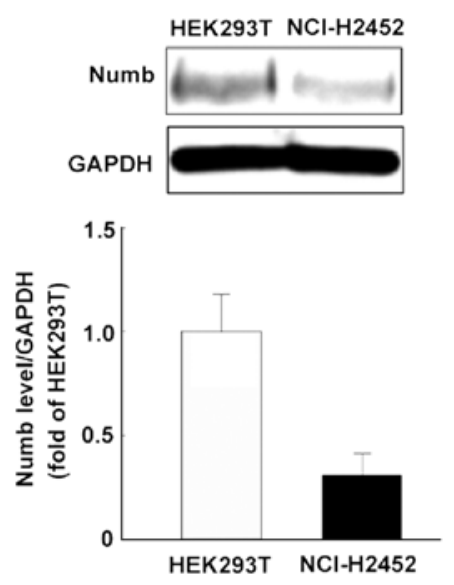

C

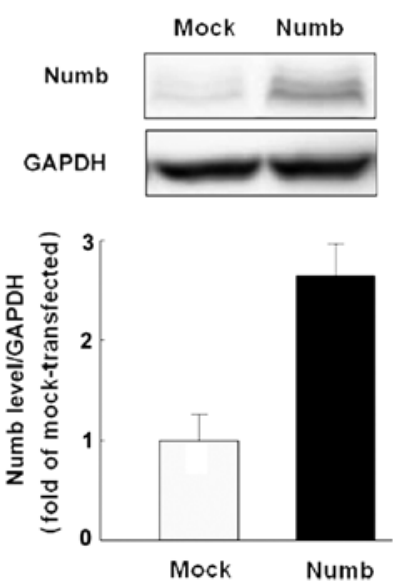

Figure 3. Expression of Numb in NCI-H2452 cells. (A and B) Before transfection, comparable levels of Numb mRNA were detected in NCI-H2452 cells when compared to HEK-293T cells, while there were markedly different protein levels of Numb between the two cell types. (C) Western blot analysis of Numb at $72 \mathrm{~h}$ after transfection. NCI-H2452 cells transfected with pcDNA3.1-Numb showed higher Numb protein expression compared with that of the control.

NCI-H2452 cells by western blot analysis. Numb-transfected cells showed increased activated caspase-3 (17 kDa) and caspase- $9(35 \mathrm{kDa})$ bands compared with the mock-transfected cells. In addition, we found release of cytochrome $c$ into the cytoplasm as well as downregulated levels of XIAP and survivin in the Numb-transfected cells (Fig. 6). 
A

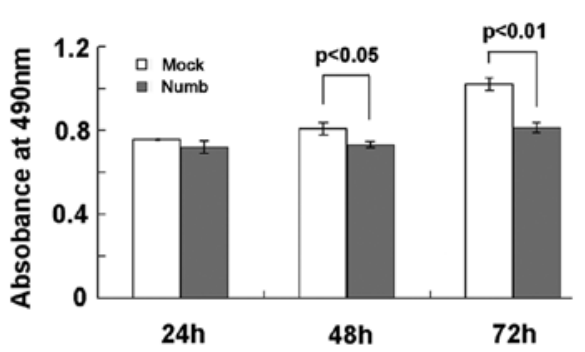

B

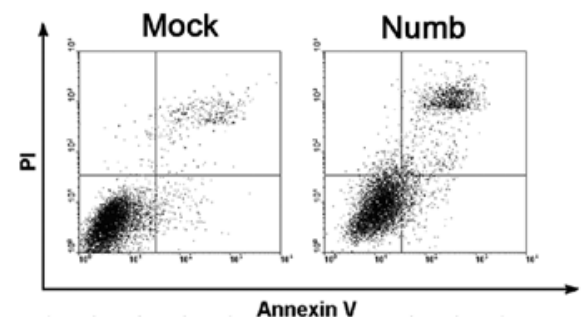

C

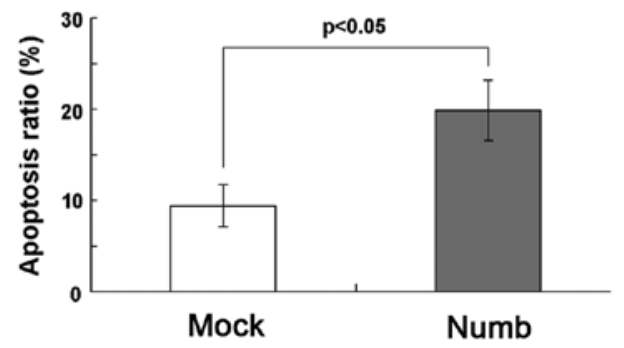

Figure 4. Effects of Numb on cell viability and apoptosis in NCI-H2452 cells. (A) The absorbance of Numb-transfected cells, as indicated by MTT, was markedly decreased at 48 and $72 \mathrm{~h}$ after transfection compared to that of the mock-transfected cells $\left({ }^{*} \mathrm{P}<0.05,{ }^{* *} \mathrm{P}<0.01\right)$. There were no significant differences between the two groups at $24 \mathrm{~h}$. (B and C) Numb induced apoptosis at $72 \mathrm{~h}$ after transfection in NCI-H2452 cells as detected by Annexin V-PI analysis. The apoptosis rate (the percentage of Annexin V/PI double-positive cells) was notably higher in Numb-transfected $(19.88 \pm 3.31 \%)$ than that in the mock-transfected cells $(9.41 \pm 2.34 \%$; $\mathrm{P}<0.05)$.

Numb sensitizes NCI-H2452 cells to cisplatin. MTT assay was used to determine the cell growth and proliferation of the NCI-H2452 cells after treatment with cisplatin for $24 \mathrm{~h}$. Significant decreases in cell viability were observed in Numbtransfected cells treated with cisplatin at concentrations of 1-10 $\mu \mathrm{mol} / \mathrm{l}$ for $24 \mathrm{~h}$ compared with the mock-transfected cells $(\mathrm{P}<0.05, \mathrm{P}<0.01 ;$ Fig. 7A). We next sought to determine whether Numb plays a role in sensitizing NCI-H2452 cells to cisplatininduced apoptosis. The apoptosis rate of the cells was assayed using flow cytometry after treatment with $5 \mu \mathrm{mol} / 1$ cisplatin for $24 \mathrm{~h}$. The percentage of early and late apoptotic cells was notably higher in the Numb-transfected cells $(32.33 \pm 5.64 \%)$ when compared with the percentage in the mock-transfected cells $(16.04 \pm 3.20 \%$; $\mathrm{P}<0.05)$ (Fig. 7B and C).

\section{Discussion}

In the present study, we reported the role of Numb as a tumor suppressor in epithelioid MPM, and revealed that it enhances the sensitivity of the human MPM cell line NCI-H2452 to cisplatin.

Our data demonstrated that Numb staining in normal pleural specimens was intense, but in tumors it was weak and in many

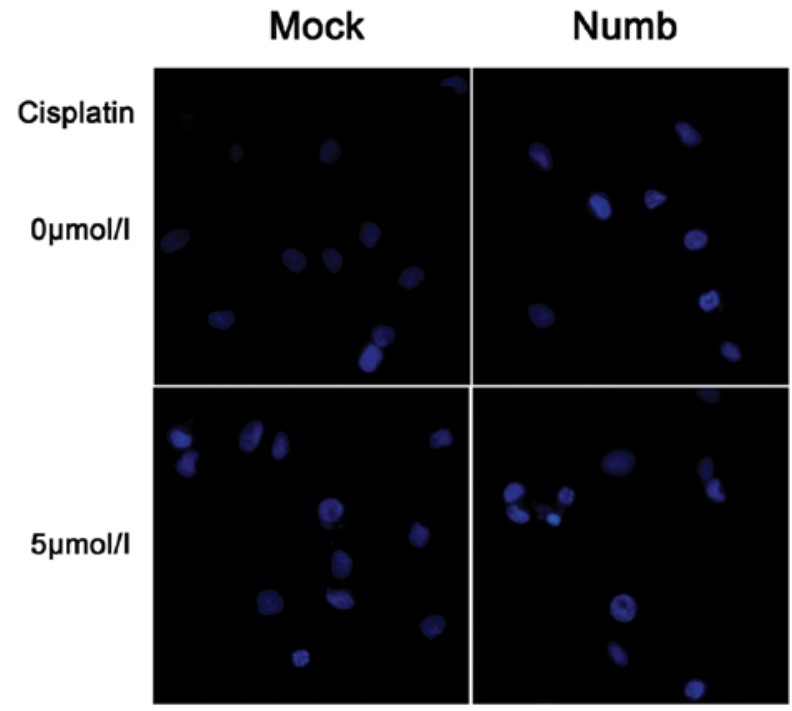

Figure 5. Morphological nuclear changes in apoptotic cells. Representative images (magnification, $\mathrm{x} 400$ ) of apoptotic cells as shown by Hoechst staining in Numb-transfected and mock-transfected cells with or without cisplatin treatment. Cells showing nuclear condensation and fragmentation are considered to be undergoing apoptosis

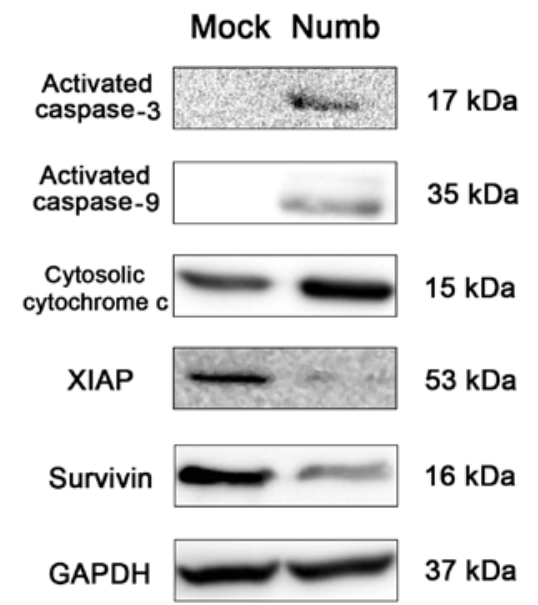

Figure 6. Western blot analysis of caspase-3, caspase-9, cytosolic cytochrome $c$ XIAP, and survivin expression in Numb-transfected and mock-transfected cells at 48-72 $\mathrm{h}$ after transfection.

cases completely absent. In accordance with the present study, the expression of Numb was found to be completely lost in breast cancers and non-small cell lung carcinomas (NSCLCs) (20,21). In addition, Numb expression was inversely correlated with the Ki-67 labeling index, an indicator of tumor aggressiveness. This finding is consistent with a study concerning breast cancer (20). High Ki-67 index is the marker of rapid cell proliferation which is related to high malignant potential, high recurrence rate and poor survival (28). This suggests that loss of Numb expression is involved in the formation and development of epithelioid MPM. Negative Numb immunohistochemistry with poor prognosis was previously reported in human salivary gland carcinomas and breast tumors $(12,24)$. Our data also showed that the reduction in Numb expression was associated with poor long term outcome in epithelioid MPM patients. Numb plays an important role in tumorigenesis 
A

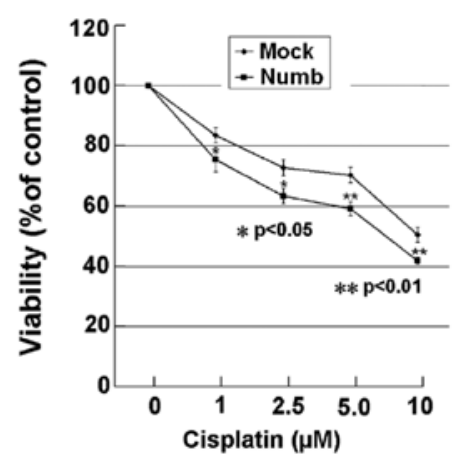

B

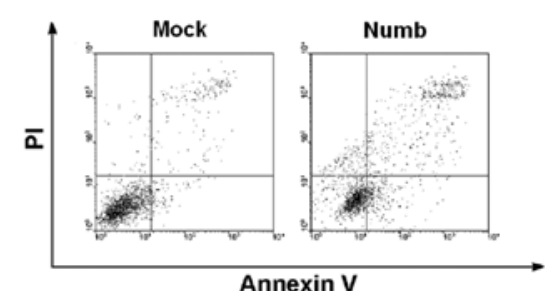

C

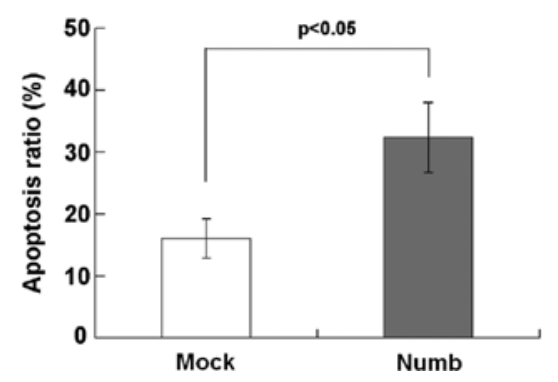

Figure 7. Effects of Numb on the sensitivity of NCI-H2452 cells to cisplatin. (A) The Numb-transfected and mock-transfected cells were treated with cisplatin at concentrations ranging from 1 to $10 \mu \mathrm{mol} / 1$ and incubated for $24 \mathrm{~h}$. The viability (\% of untreated cells) was lower in the Numb-transfected cells when compared with the mock-transfected cells. (B and C) The apoptosis rate (the percentage of Annexin V/PI double-positive cells) after a 24-h treatment of $5 \mu \mathrm{mol} / 1$ cisplatin was notably higher in the Numb-transfected cells $(32.33 \pm 5.64 \%)$ when compared with that of the mock-transfected cells $(16.04 \pm 3.20 \%, \mathrm{P}<0.05)$.

due to its crucial feature of asymmetric cellular distribution. Moreover, due to its ability to function as an adaptor for E3-enzymes, Numb is involved in a complex network of ubiquitin-regulated pathways which are related to the development of cancer. Numb does not live up to its name as 'fate determinant'.

We selected NCI-H2452, an epithelioid human MPM cell line to investigate the expression and function of Numb in vitro. We detected comparable levels of Numb mRNA in NCI-H2452 cells vs. HEK-293T cells, and this contrasted with the markedly different levels of Numb protein in the same cultures. Thus, we speculated that loss of Numb expression in NCI-H2452 cells is determined at the posttranslational level, through enhanced protein degradation, similarly to what was shown in breast cancers (20) and NSCLCs (21), but the exact mechanism needs to be further explored.

In Numb-negative breast tumor cells, re-expression of Numb was found to selectively suppress growth (20). Our results also showed that overexpression of Numb inhibits proliferation and promotes apoptosis in NCI-H2452 cells.
Malignant cells are frequently resistant to apoptotic stimuli, and the inhibition of apoptosis may enhance tumor development. Caspase- 3 activity is often used as a definite marker for apoptosis, and cytochrome $c /$ caspase-9 activation is an initial event in the intrinsic pathway for apoptosis. In the present study, activated caspase- 3 and caspase- 9 bands as well as release of cytochrome $c$ to the cytoplasm were observed in Numb-transfected cells. This suggests that overexpression of Numb induced apoptosis perhaps through cytochrome $c /$ caspase-9/caspase-3 signaling. Inhibitors of apoptosis proteins, IAPs, are a family of proteins that regulate the cytochrome c/caspase activating pathway (29). XIAP is involved in the inactivation of caspase- $3,-7$ and -9 and has been found to be elevated in mesothelioma $(30,31)$. Several studies have now indicated that higher levels of XIAP may contribute to chemotherapy resistance in mesothelioma cells $(32,33)$. Survivin was also found actively expressed in MPM $(32,34)$ and it has an important role in maintaining apoptosis resistance. It can partially block activation of caspases due to its ability to bind to activated caspases $(35,36)$. Survivin inhibition increases the rates of both spontaneous and radiation-induced apoptosis (37) as well as decreased survival of MPM cells (38). In our study, Numb overexpression decreased the expression of XIAP and survivin, yet the mechanism in unknown. It has been shown that IAPs contain a RING finger domain which confers ubiquitin protease ligase (E3) activity, and these IAPs having E3 activity are able to catalyze their own ubiquitination and degradation. As we know, Numb is at the intersection of a complex network of E3-ligases for it contains a functional phosphotyrosine-binding (PTB) domain. We hypothesized that Numb downregulates IAPs directly through promoting ubiquitination and protease degradation, but further study is needed to explore the exact mechanism. However, we speculate that IAPs play important roles in the apoptosis promoted by Numb. Overexpression of Numb to downregulate IAPs may be an important strategy for overcoming chemotherapy and apoptosis resistance in MPM.

The standard first-line treatment used for MPM is cisplatin-based chemotherapy, either as a single agent or in combination with pemetrexed. Its cytotoxicity is mediated through platinum-DNA adducts and subsequent induction of apoptosis (39). However, in MPM the chemotherapy resistance leads to low efficacy and high doses of cisplatin cause prohibitive toxicity such as nephrotoxicity (40). In the present study, we showed that overexpression of Numb significantly enhanced sensitivity to cisplatin-induced apoptosis and suppressed growth in NCI-H2452 cells. These data indicate that Numb may be involved in conferring cisplatin sensitivity to MPM cells. Decreased caspase- 3 and caspase- 9 activation have been described as an additional mechanism of cisplatin resistance in various types of cancers (41-44). To confirm whether or not Numb sensitizes MPM cells to cisplatin through the caspasedependent apoptotic pathway, further investigation is needed.

In conclusion, our results revealed that Numb was frequently downregulated in epithelioid MPM cases, which was also associated with poor prognosis. Overexpression of Numb in NCI-H2452 cells inhibited proliferation and promoted apoptosis, and we speculated that cytochrome $c /$ caspase signaling was a possible mechanism through which Numb enhanced apoptosis. Moreover, Numb sensitized NCI-H2452 cells to 
cisplatin. These results have important implications for Numb in the development of novel strategies for drug-resistant MPM therapy, and further investigation is warranted.

\section{Acknowledgements}

We thank the doctors from the Department of Respiratory Medicine and the Department of Pathology of Shandong Provincial Hospital for their technical support.

\section{References}

1. Peto J, Decarli A, La Vecchia C, Levi F and Negri E: The European mesothelioma epidemic. Br J Cancer 79: 666-672, 1999.

2. Bianchi $\mathrm{C}$ and Bianchi T: Malignant mesothelioma: global incidence and relationship with asbestos. Ind Health 45: 379-387, 2007.

3. Robinson BWS, Musk AW and Lake RA: Malignant mesothelioma. Lancet 366: 397-408, 2005.

4. Beachy PA, Karhadkar SS and Berman DM: Tissue repair and stem cell renewal in carcinogenesis. Nature 432: 324-331, 2004

5. Uemura T, Shepherd S, Ackerman L, Jan LY and Jan YN: numb, a gene required in determination of cell fate during sensory organ formation in Drosophila embryos. Cell 58: 349-360, 1989.

6. Rhyu MS, Jan LY and Jan YN: Asymmetric distribution of numb protein during division of the sensory organ precursor cell confers distinct fates to daughter cells. Cell 76: 477-491, 1994.

7. Morrison SJ and Spradling AC: Stem cells and niches: mechanisms that promote stem cell maintenance throughout life. Cell 132: 598-611, 2008

8. Rašin MR, Gazula VR, Breunig JJ, et al: Numb and Numb are required for maintenance of cadherin-based adhesion and polarity of neural progenitors. Nat Neurosci 10: 819-827, 2007.

9. Nishimura $T$ and Kaibuchi K: Numb controls integrin endocytosis for directional cell migration with aPKC and PAR-3. Dev Cell 13: 15-28, 2007.

10. McGill MA and McGlade CJ: Mammalian numb proteins promote Notch1 receptor ubiquitination and degradation of the Notch1 intracellular domain. J Biol Chem 278: 23196-23203, 2003.

11. Di Marcotullio L,Ferretti E, Greco A, et al: Numb is a suppressor of Hedgehog signalling and targets Gli1 for Itch-dependent ubiquitination. Nat Cell Biol 8: 1415-1423, 2006.

12. Colaluca IN, Tosoni D, Nuciforo P, et al: NUMB controls p53 tumour suppressor activity. Nature 451: 76-80, 2008.

13. Pece S, Confalonieri S, Romano PR and Di Fiore PP: NUMB-ing down cancer by more than just a NOTCH. Biochim Biophys Acta 1815: 26-43, 2011.

14. Singh A and Settleman J: EMT, cancer stem cells and drug resistance: an emerging axis of evil in the war on cancer. Oncogene 29: 4741-4751, 2010.

15. Thiery JP, Acloque H, Huang RYJ and Nieto MA: Epithelialmesenchymal transitions in development and disease. Cell 139: 871-890, 2009

16. Lanzetti L and Di Fiore PP: Endocytosis and cancer: an 'insider' network with dangerous liaisons. Traffic 9: 2011-2021, 2008.

17. Vaccari T and Bilder D: At the crossroads of polarity, proliferation and apoptosis: the use of Drosophila to unravel the multifaceted role of endocytosis in tumor suppression. Mol Oncol 3: 354-365, 2009.

18. Goldstein B and Macara IG: The PAR proteins: fundamental players in animal cell polarization. Dev Cell 13: 609-622, 2007.

19. Aranda V, Nolan ME and Muthuswamy SK: Par complex in cancer: a regulator of normal cell polarity joins the dark side. Oncogene 27: 6878-6887, 2008.

20. Pece S, Serresi M, Santolini E, et al: Loss of negative regulation by Numb over Notch is relevant to human breast carcinogenesis J Cell Biol 167: 215-221, 2004
21. Westhoff B, Colaluca IN, D'Ario G, et al: Alterations of the Notch pathway in lung cancer. Proc Natl Acad Sci USA 106: 22293-22298, 2009.

22. Caussinus E and Gonzalez C: Induction of tumor growth by altered stem-cell asymmetric division in Drosophila melanogaster. Nat Genet 37: 1125-1129, 2005.

23. Bric A, Miething C, Bialucha CU, et al: Functional identification of tumor-suppressor genes through an in vivo RNA interference screen in a mouse lymphoma model. Cancer Cell 16: 324-335, 2009.

24. Maiorano E, Favia G, Pece S, et al: Prognostic implications of NUMB immunoreactivity in salivary gland carcinomas. Int J Immunopathol Pharmacol 20: 779-789, 2007.

25. Marino S: Medulloblastoma: developmental mechanisms out of control. Trends Mol Med 11: 17-22, 2005.

26. Yan B, Omar FM, Das K, et al: Characterization of Numb expression in astrocytomas. Neuropathology 28: 479-484, 2008.

27. Travis W, Brambilla E, Muller-Hermelink H and Harris C (eds): World Health Organization Classification of Tumours. Pathology \& Genetics. Tumours of the Lung, Pleura, Thymus and Heart. IARC Press, Lyon, 2004

28. Heidebrecht H, Buck F, Endl E, Kruse M, Adam K and Andresen K: Ki-67-Mcm6, a new MoAb specific to Mcm6: comparison of the distribution profile of Mcm6 and Ki-67 antigen. Lab Invest 81: 1163-1165, 2008

29. Adams JM and Cory S: The Bcl-2 protein family: arbiters of cell survival. Science 281: 1322-1326, 1998.

30. Kleinberg L, Lie AK, Flørenes VA, Nesland JM and Davidson B: Expression of inhibitor-of-apoptosis protein family members in malignant mesothelioma. Hum Pathol 38: 986-994, 2007.

31. Wu M, Yuan S, Szporn AH, Gan L, Shtilbans V and Burstein DE: Immunocytochemical detection of XIAP in body cavity effusions and washes. Mod Pathol 18: 1618-1622, 2005.

32. Gordon G, Mani M, Mukhopadhyay L, et al: Expression patterns of inhibitor of apoptosis proteins in malignant pleural mesothelioma. J Pathol 211: 447-454, 2007.

33. Gordon G, Mani M, Mukhopadhyay L, et al: Inhibitor of apoptosis proteins are regulated by tumour necrosis factor- $\alpha$ in malignant pleural mesothelioma. J Pathol 211: 439-446, 2007.

34. Hmeljak J, Erčulj N, Dolžan V, Kern I and Cör A: BIRC5 promoter SNPs do not affect nuclear survivin expression and survival of malignant pleural mesothelioma patients. J Cancer Res Clin Oncol 137: 1641-1651, 2011.

35. Tamm I, Wang Y, Sausville E, et al: IAP-family protein survivin inhibits caspase activity and apoptosis induced by Fas (CD95), Bax, caspases, and anticancer drugs. Cancer Res 58: 5315-5320, 1998.

36. Kobayashi K, Hatano M, Otaki M, Ogasawara T and Tokuhisa T: Expression of a murine homologue of the inhibitor of apoptosis protein is related to cell proliferation. Proc Natl Acad Sci USA 96: 1457-1462, 1999

37. Kim KW, Mutter RW, Willey CD, et al: Inhibition of survivin and aurora B kinase sensitizes mesothelioma cells by enhancing mitotic arrests. Int J Radiat Oncol Biol Phys 67: 1519-1525, 2007.

38. Xia C, Xu Z, Yuan X, et al: Induction of apoptosis in mesothelioma cells by antisurvivin oligonucleotides. Mol Cancer Ther 1: 687-694, 2002.

39. Rabik CA and Dolan ME: Molecular mechanisms of resistance and toxicity associated with platinating agents. Cancer Treat Rev 33: 9-23, 2007.

40. Boulikas T and Vougiouka M: Cisplatin and platinum drugs at the molecular level (Review). Oncol Rep 10: 663-682, 2003.

41. Ikuta K, Takemura K, Kihara M, et al: Defects in apoptotic signal transduction in cisplatin-resistant non-small cell lung cancer cells. Oncol Rep 13: 1229-1234, 2005.

42. Okouoyo S, Herzer K, Ucur E, et al: Rescue of death receptor and mitochondrial apoptosis signaling in resistant human NSCLC in vivo. Int J Cancer 108: 580-587, 2003

43. Yang X, Zheng F, Xing H, et al: Resistance to chemotherapyinduced apoptosis via decreased caspase-3 activity and overexpression of antiapoptotic proteins in ovarian cancer. $\mathrm{J}$ Cancer Res Clin Oncol 130: 423-428, 2004.

44. Mueller T, Voigt W, Simon H, et al: Failure of activation of caspase-9 induces a higher threshold for apoptosis and cisplatin resistance in testicular cancer. Cancer Res 63: 513-521, 2003. 